\title{
Optimization of IEEE 802.11 MAC Protocol
}

\author{
Vikas Pachisia $^{1}$, Annappa B ${ }^{2}$ \\ ${ }^{1,2}$ Department of Computer Engineering, National Institute of Technology Karnataka, Srinivasnagar P.O. 575025, Surathkal, Karnataka, \\ India
}

\begin{abstract}
As of today wireless technologies are not up to the mark in providing good communication services compared to wired devices. This fact is truer in the field of data communications wherein the wired devices provide highly reliable and fast data transfer rates compared to wireless devices. In the past, some major work is already carried out by various authors in this segment. This paper is an effort in continuing to bring the change by modifying the already proven concepts and bringing in new concepts. To this extent, we are trying to improve the protocol capacity of the IEEE $802.11 \mathrm{MAC}$ protocol in three different ways.
\end{abstract}

Keywords: Contention Window, Back off time, Poisson distribution, IEEE 802.11 MAC, DCF, RTS/CTS, CSMA/CA

CR Subject Classification: G.m.0 Queuing theory, G.3.2 Statistical computing, Wireless Local Networks access scheme

AMS: 62H12 Estimation

\section{Introduction}

From the time wireless technology was commercialized, scientists and industrialists had a strong feeling of its success in the market. There is no doubt that wireless communication will be the future technology that will prevail in every aspect of human life. Unbelievable, but wireless technology had a great success and failure. The wireless voice communication performed very well and is continuing to perform well in the market. However, the consumer base did not respond to the wireless data communication technology as expected. The reason can partially be attributed to the non-availability of the technology that can provide services on par with wired LANs. But a major factor for its non-success, we feel, is it does not keep up the promise of performing up to the extent that it claims. That is, an IEEE 802.11 [7] complaint device does not provide good data transfer rate under high network loads. Its performance degrades heavily beyond ten nodes.

The above factor's warrants the need for research in this field to find a way to maximally utilize the available resources. We followed various concepts [1] [2] [3] [4] in studying the protocol in a different perspective. For the analytical model in our paper, we modeled our system of WLAN as poisson distributed [5].

\section{Approach}

Method I: Here we focus on the analytical way of finding an optimal contention window size. A set of wireless devices forming a wireless local area network can be inherently modeled as poisson distributed. The single shared medium (wireless channel) is viewed as a processor, to which the packets from different wireless devices are queued for transmission to destinations. Each wireless device will have its own output queue and hence the processor has a distributed queue. Thus the job of the processor is to transmit the packet from source to destination taking packets from the distributed queue. The packet sizes themselves are taken from Poisson distribution. In this process it may so happen that, more than one wireless device put a packet in the front of the queue for transmission at the same time. This will lead into the collision of the packets and hence an unsuccessful transmission attempt. In this manner a packet may undergo multiple collisions before being successfully transmitted. The time interval between two consecutive successful packet transmissions is known as the virtual transmission time. The tree structure of figure 1 will explain the various times involved in a virtual transmission time.

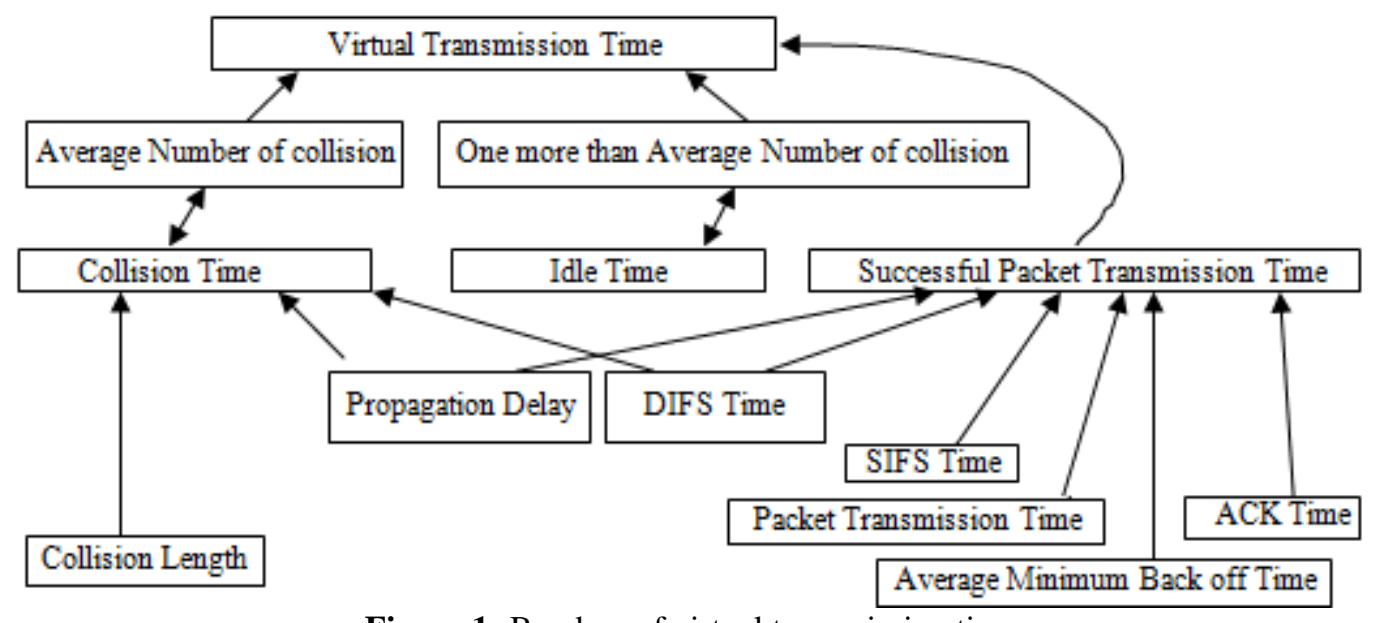

Figure 1: Breakup of virtual transmission time

Volume 9 Issue 3, March 2020 www.ijsr.net

Licensed Under Creative Commons Attribution CC BY 


\section{International Journal of Science and Research (IJSR) \\ ISSN: 2319-7064}

ResearchGate Impact Factor (2018): 0.28 | SJIF (2019): 7.583

A simple convention can be followed to interpret figure 1 . The two objects (text boxes) connected by an arrow with double head is to be multiplied. For an arrow with single head, the object present at the tail of the arrow is to be added into the object present at the head of the arrow.

The parameters that are assumed to be fixed (as they are dependent on the physical channel) are Propagation delay, SIFS time, DIFS time, ACK time (ACK length is fixed), Average minimum back off time. Thus, the elements to be computed are Average number of collision, Collision Length, Idle Time. These values are found as shown below:

Each transmission attempt represents a contention cycle. A virtual transmission time is made up of multiple contention cycles. We also make an assumption that the wireless channel always has a packet ready for transmission. Since we model the WLAN environment as Poisson distribution, all wireless devices will be giving packets to the channel for transmission according to this distribution. Thus probability that $\mathrm{k}$ packets will arrive for transmission during a contention cycle is:

$$
\mathrm{P}(\text { packets }=\mathrm{k})=\mathrm{e}^{\alpha \mathrm{m}}\left(\alpha_{\mathrm{m}}\right)^{\mathrm{k} / \mathrm{k}}
$$

Where $\alpha_{\mathrm{m}}=\boldsymbol{M}$ (No. of wireless devices) x probability of a device transmitting in this contention cycle.

Probability (device transmitting in a contention cycle) $=2$ / $(\mathrm{E}[\mathrm{cw}]+1)(2)$

Next we find out the probability of collision as given by (3). This equation will be used to find the probability of different contention window sizes at each contention cycle (This probability is specified in (5)). The second probability is based on the number of collision a packet undergoes before being successfully transmitted. For this, we need to find the probability of a packet undergoing ' $n$ ' collision before a successful transmission, which is given by (4).

$$
\begin{aligned}
& \mathrm{P}_{\text {coll }}=1-\left(\mathrm{e}^{-\mathrm{Ma} a}+\mathrm{M} \alpha \mathrm{e}^{-\mathrm{M} a}\right) \\
& P_{\text {Ncoll }}(n)=e^{-P_{\text {coll }}}\left(P_{\text {coll }}{ }^{n}\right) / n ! \\
& \mathrm{P}\left\{\mathrm{CW}=\mathrm{CW}_{\mathrm{i}}\right\}=\left\{\begin{array}{cl}
(1-\alpha) & \mathrm{J}=0 \\
\mathrm{j} & \\
(1-\alpha)\left[1-\mathrm{e}^{-\alpha}\left(\sum \alpha^{\mathrm{k}} / \mathrm{k} !\right)\right] & \mathrm{J}=1,2,3,4,5 \\
\mathrm{~K}=0 &
\end{array}\right.
\end{aligned}
$$

Where $\mathrm{CW}_{0}=8, \mathrm{CW}_{1}=16, \mathrm{CW}_{2}=32, \mathrm{CW}_{3}=64, \mathrm{CW}_{4}=128$, $\mathrm{CW}_{5}=256$

Next we propose the expression for idle time and number of collision, which will help us in finding the optimal contention window size for a given number of wireless devices, a given packet size and $\alpha$

$$
\begin{aligned}
& \mathrm{E}[\mathrm{Idle}]=\mathrm{e}^{-\mathrm{M}[a /} /\left(1-\mathrm{e}^{-\mathrm{M} \sigma a}\right) \\
& \mathrm{E}\left[\mathrm{N}_{\mathrm{c}}\right]=\left(\left(1-\mathrm{e}^{-\mathrm{M} \sigma a}\right) /\left(\mathrm{M} \alpha \mathrm{e}^{-\mathrm{M} a}\right)\right)-1
\end{aligned}
$$

Since the expression for calculating collision length is very complex and is not feasible at run time, we make an assumption of the system performing best in steady state condition, wherein the average idle time and the collision time should be same. This leads us to the expression for collision length in terms of idle time and average number of collisions as given in (8).

$\mathrm{E}\left[\right.$ Collision length] $=\left\{\left(\mathrm{E}\left[\mathrm{N}_{\mathrm{c}}\right]+1\right) / \mathrm{E}\left[\mathrm{N}_{\mathrm{c}}\right]\right\}\{\mathrm{E}[\mathrm{Idle}]\}(8)$
The above equations help us in finding the appropriate contention window size for various network configurations as given in table 1. The major elements of the network configuration which affect the choice of contention window size are the number of stations in the network and the length of the packets that they transmit. Finally, we can find the theoretical capacity of the protocol that can be attained by the proper selection of the contention window size. The protocol capacity equation is given by (9).

$$
\rho_{\max }=\mathrm{PS}_{\mathrm{avg}} / \mathrm{T}_{\mathrm{v}} \text { (Virtual Transmission time) }
$$

Where $\mathrm{PS}_{\mathrm{avg}}=\mathrm{T}_{\text {slot }} / \lambda, \lambda$ being the parameter of Poisson distribution for packet size and Tslot is the length of the slot in time units.

\section{Results}

The table below shows results for a few network configurations. This table also lists the optimal contention window sizes for the given network and its theoretical protocol capacity.

Table 1: Optimal $\alpha$, contention window size, protocol capacity for various network configurations

\begin{tabular}{|c|c|c|c|c|c|}
\hline $\begin{array}{c}\text { Stations } \\
(\mathrm{M})\end{array}$ & $\begin{array}{c}\text { Packet } \\
\text { Size }(\lambda)\end{array}$ & $\mathrm{E}[\alpha]$ & $\mathrm{E}[\mathrm{CW}]$ & $\mathrm{T}_{\text {slot }}$ & $\rho_{\max }$ \\
\hline 500 & 100 & 0.0161302 & 122.99102305 & 0.00002 & 0.00026155334 \\
\hline 100 & 100 & 0.0790924 & 24.28687965 & 0.00002 & 0.00010464 \\
\hline 50 & 100 & 0.1590444 & 11.575104814 & 0.00002 & 0.0001046401 \\
\hline 20 & 100 & 0.233007 & 7.583433116 & 0.00002 & 0.00010367150 \\
\hline
\end{tabular}

Mathematical model given above represents one of the methods of studying the protocol capacity. The other two methods are briefly described below. These works will be over by the end of this month.

Method II: When a wireless device gets access to the channel for the first time, it is allowed to access the channel for some maximum number of times consecutively. The wireless device gets a back off time of one slot, till the number of successful packet transmission reaches threshold value after which it takes very high back off time.

Method III: When the wireless channel is free for some threshold time limit, then instead of decrementing the back off slots by 1 unit we decrement it $\mathrm{x}$ units where $\mathrm{x}$ depends on the network configuration.

\section{Conclusion}

This paper gives the analytical limit for the IEEE 802.11 MAC protocol capacity. Also it presents two other methods as an attempt to improve the channel capacity. The results for some of the network configuration show that the IEEE 802.11 protocol can not reach a maximum capacity of 1 . It is also supported by the general theory, to avoid collision the network should have a large average back off time and to avoid high delay the network should have a low average back off time. Hence, optimizing the protocol capacity involves a trade off between low collisions and low idle time. 


\section{Acknowledgements}

We would like to thank Narsimha babu, Younggoo Kwon et. al, Hui Ma et. al, Cali et. al for their support extended to me. We also thank Dr. K.C. Shet, Dr. K. Chandrasekaran and the department for providing me the facilities to carry out the project in the department.

We wish to thank the anonymous referees for their careful reading of the manuscript and their fruitful comments and suggestions.

\section{References}

[1] Younggoo Kwon, Yuguang Fang, and Haniph Latchman, "Design of MAC Protocols With Fast Collision Resolution for Wireless Local Area Networks", IEEE Transactions On Wireless Communications, 3(3), 793-807(2004).

[2] F. Cali, M. Conti, and E. Gregori, "Dynamic tuning of the IEEE 802.11 protocol to achieve a theoretical throughput limit," IEEE/ACM Trans. Network., 8(6), 785-799(2000).

[3] Hui Ma, Xing Li, Hewu Li, Peiyun Zhang, Shixin Luo, Cong Yuan, "Dynamic optimization of IEEE 802.11 CSMA/CA based on the number of competing stations", Communications, 2004 IEEE International Conference on , Volume: 1, 20-24 June 2004 Pages:191 - 195

[4] V. Bharghavan, "MACAW: A media access protocol for wireless LAN's," in Proc. SIGCOMM'94, London, U.K., Aug. 1994, pp. 212-225

[5] Kishore Shridharbhai Trivedi, "Probability and Statistics with Reliability, Queuing, and Computer Science Applications", John Wiley and Sons, New York, 2001

[6] Kaveh Pahlavan, Prashant Krishnamurthy, "Principles of Wireless Networks - A unified approach", Pearson Prentice Hall PTR, 2002.

[7] IEEE P802.11. Standard for Wireless LAN Medium Access Control (MAC) and Physical Layer (PHY) Specifications, 1997.

[8] IEEE standard for Wireless LAN Medium Access Control (MAC) and Physical Layer (PHY) specifications, ISO/IEC 8802-11:1999(E), Aug. 1999

[9] IEEE P802.11b. Supplement to Standard IEEE 802.11, Higher speed Physical Layer (PHY) extension in the 2.4GHz band, 1999 .

[10] VINT Group, "UCB/LBNL/VINT network simulator ns(version 2)," http://www.isi.edu/nsnam/ns

Volume 9 Issue 3, March 2020 www.ijsr.net 